\title{
Giant adrenal myelolipoma presenting as an incidentaloma: a case report and review of literature
}

\begin{abstract}
Adrenal Myelolipoma is a rare nonfunctional adrenal tumor usually detected incidentally on imaging done for un-related cause or due to compressive symptoms or rarely due to adrenal hormone hyper secretion or malignant transformation. A case of 35years old male was complained with non-specific pain in right thigh for one month duration without history of trauma, musculoskeletal or neurological disorder. The physical examination was un-remarkable except for systemic hypertension and a huge intra-abdominal mass in the right hypo chondrium found, without any complaints. Ultrasound and subsequently CT scan abdomen showed a large well-circumscribed mass arising from the right adrenal gland, measuring $18 \times 13 \times 18 \mathrm{~cm}$. The adrenal mass was detected incidentally. The patient with large tumors may have some compressive symptoms. Very rarely a giant tumor is completely asymptomatic and detected incidentally during work-up for the other un-related complain. Herein we describe a case of a young male with giant adrenal myelolipoma detected incidentally. Therefore, it is very significant to diagnose carefully for the planning of therapeutic management.
\end{abstract}

Keywords: adrenal myelolipoma, tumor, ultrasound, ct-scan, histopathology, immunostaining

\author{
Volume 4 Issue 2 - 2017
}

\author{
Gyan Chand,' Adarsh Ranjan, ${ }^{2}$ Ashok Kumar, ${ }^{3}$ \\ Sudhi Agarwal, ${ }^{4}$ Amit Agarwal, ${ }^{5}$ Ram Nawal \\ Rao, ${ }^{6}$ Hira Lal ${ }^{7}$ \\ 'Additional Professor, Department of Endocrine Surgery, Sanjay \\ Gandhi Postgraduate Institute of Medical Sciences, India \\ ${ }^{2}$ Demonstrator, Department of Anatomy, Hind Institute of \\ Medical Sciences, India \\ ${ }^{3}$ Department of Genetics, Sanjay Gandhi Postgraduate Institute \\ of Medical Sciences, India \\ ${ }^{4}$ Senior Resident, Department of Endocrine Surgery, Sanjay \\ Gandhi Postgraduate Institute of Medical Sciences, India \\ ${ }^{5}$ Professor, Department of Endocrine Surgery, Sanjay Gandhi \\ Postgraduate Institute of Medical Sciences, India \\ ${ }^{6}$ Additional Professor, Department of Pathology, Sanjay Gandhi \\ Postgraduate Institute of Medical Sciences, India \\ ${ }^{7}$ Additional Professor, Department of Radiodiagnosis, Sanjay \\ Gandhi Postgraduate Institute of Medical Sciences, India
}

Correspondence: Gyan Chand,Associate Professor, Department of Endocrine Surgery, Sanjay Gandhi Post Graduate Institute of Medical Sciences, Lucknow, India, Tel 91522249 4409, 9194 5।54 6353(M), Fax 9152 22668777.

Email drgyanchandpgi@gmail.com

Received: May 05, 2017 | Published: August II, 2017

\section{Introduction}

Adrenal myelolipoma is relatively rare, non-functioning, benign tumor, composed of mature adipose tissue and active hematopoietic elements. These hematopoietic elements are mixture of myeloid and erythroid cells. Generally myelolipomas occurs in adrenal gland, but it can be present in other sites also. In 1905, it was initially reported by Gierke and afterword's Oberling coined the term "myelolipoma" in 1929. ${ }^{1,2}$ According to the available literature, Myelolipomas account for approximately 3 to $5 \%$ of all primary adrenal tumors. ${ }^{3}$ Even though the incidence of these tumors are mysterious, it is belief to be between $0.08 \%$ and $0.4 \%$, both gender appear to be equally affected by these tumor which are commonly found between the 50 to 70 age group. ${ }^{4}$ According to Brogna; the incidence in various series range from $0.01-0.2 \%$, however with more sophisticated imaging available, the incidence of incidentally detected myelolipomas have been increased to $10 \%$ as reported in the mid- $1990 \mathrm{~s}^{5}{ }^{5}$ The incidentally detected tumors are small in size and asymptomatic, whereas giant tumors are large in size and symptomatic. Very few cases in literature have reported cases with truly asymptomatic giant adrenal myelolipoma incidentally detected during imaging for other un-related illnesses. ${ }^{6}$ Here we have reported a case of incidentally detected giant adrenal myelolipoma, its clinical implications and a brief review of literature.

\section{Case report}

A case of 35 years old male was referred us for evaluation of a non-specific pain in right thigh for one month duration without any history of trauma, musculoskeletal or neurological disorder. The physical examination was un-remarkable except for systemic hypertension (BP-160/100mm $\mathrm{Hg}$ ) without postural drop and a huge intra-abdominal mass in the right hypochondrium, without any complaints. There is no history of abdominal and urinary complaints, weight loss, hypercortisolism, hypercatecholaminism or hyperandrogenism. His family history was non-contributory. Ultrasound and subsequently CT scan abdomen showed a large wellcircumscribed mass arising from the right adrenal gland, measuring $18 \times 13 \times 18 \mathrm{~cm}$. The mass was isodense to hypodense with areas of fat density (HU-110) and heterogeneous contrast enhancement with a thin capsule. The right kidney, was significantly displaced downwards (Figure 1). The contralateral adrenal gland was normal (Figure 2). The hormonal evaluation revealed suppressible 8:00 AM serum cortisol level $(51.1 \mathrm{nmol} / \mathrm{L})$ with $1 \mathrm{mg}$ of dexamethasone at $11 \mathrm{PM}$, normal 24hour urinary metanephrine $(176 \mu \mathrm{g} / 24 \mathrm{hour})$ and nor-metanephrine $(490 \mu \mathrm{g} / 24 \mathrm{hr})$, [the reference range being 300 and $600 \mu \mathrm{g} / 24 \mathrm{hour}$ respectively], and normal serum potassium which confirmed it to be a non-functional adrenal mass. After clinical diagnosis of nonfunctioning large right adrenal myelolipoma, he was taken up for open trans-peritoneal right adrenalectomy. Per-operatively a lobulated, well encapsulated right adrenal mass $24 \times 15 \times 12 \mathrm{~cm}$, weighing $2000 \mathrm{~g}$ was excised, the cut surface showed predominantly fatty component with few area of hemorrhage (Figure 3). There was no significant blood pressure fluctuation during surgery and the post-op recovery was un-eventful. The blood pressure improved following surgery and he remained normotensive without any anti-hypertensive drug. The histopathology showed an encapsulated tumor comprising 
predominantly of mature adipocytes with intervening areas of hematopoietic cells which are comprising of myeloid cells, erythroid cells and occasional megakaryocytes and a portion of normal adrenal at the periphery (Figure 4).

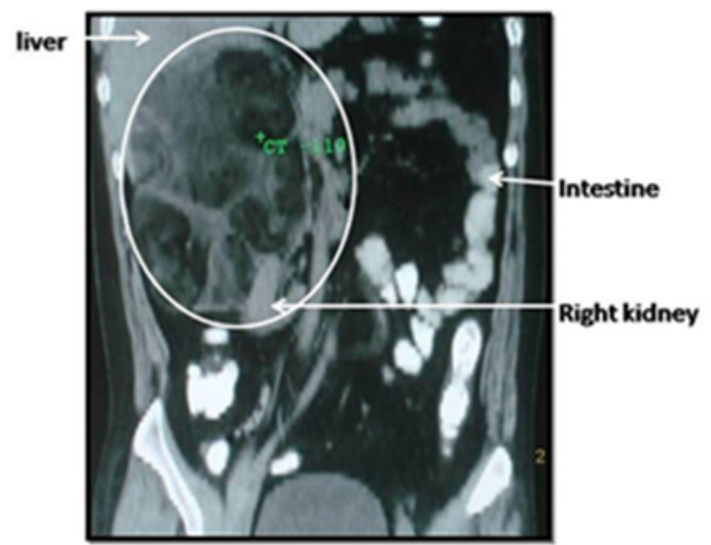

Figure I Representation of CT-Scan image of coronal section of Abdomen after giving oral contrast of gastrographin. Oval circle represents suprarenal mass with heterogeneous density having hounsfield unit is- II0.

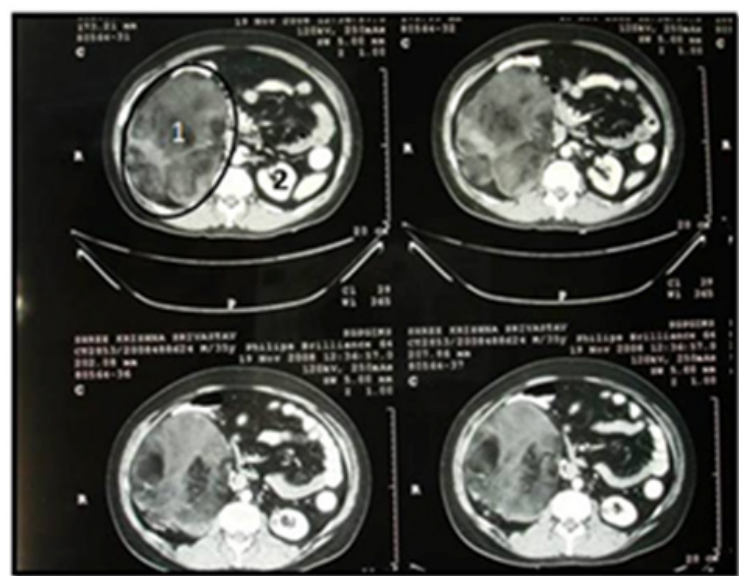

Figure 2 Representation of CT scan of $5 \mathrm{~mm}$ axial cut section of abdomen after giving intravenous injection of ultravist contrast media. Black circle represents the heterogeneous densities with well-defined margin shows suprarenal mass. I, suprarenal gland; 2, left kidney.

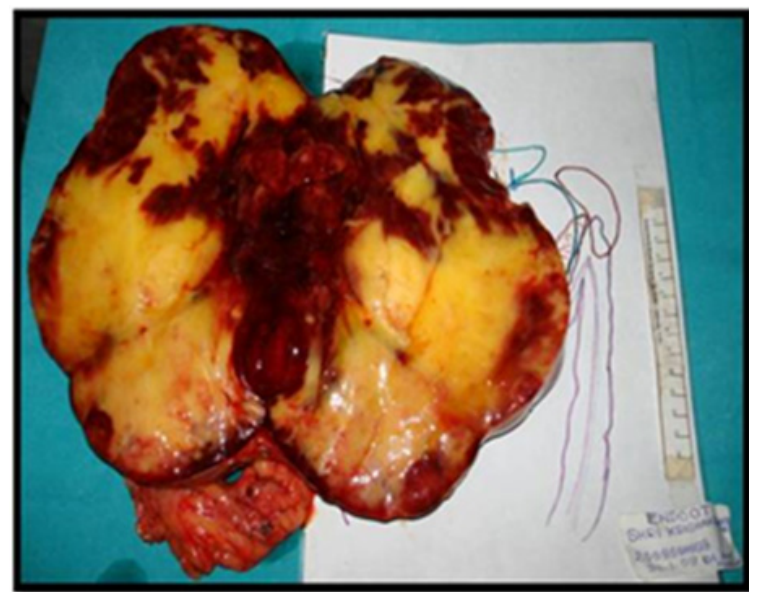

Figure 3 Represent Cut section of the mass showing the large yellowish area with area of hemorrhagic necrosis.
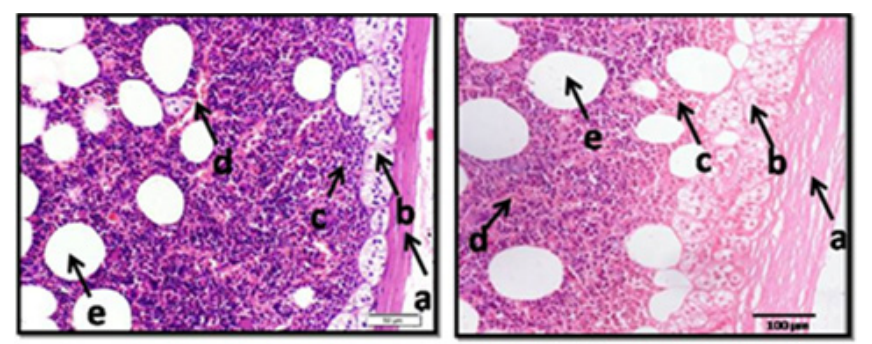

Figure $4 \mathrm{H} \& \mathrm{E}$ stained histo-micrograph of adrenal tumor showing encapsulated tumor comprising predominantly of mature adipocytes with intervening areas of hematopoietic cells comprising of myeloid cells, erythroid cells and occasional megakaryocytes and a portion of normal adrenal at the periphery (H \& E 200 X). a-capsule; b-zona glomerulosa; c-zonafasciculata; d-adrenocortical sinusoids; e-adipocyte.

\section{Discussion}

Adrenal myelolipoma are infrequent, nonmalignant tumor composed of matured adipose tissue and hematopoietic elements (mixture of myeloid and erythroid cells). The most common site of myelolipoma is adrenal gland but it is infrequently present in the paravertebral regionas, retroperitoneum, pelvis, and mediastinum, as an isolated soft tissue mass. ${ }^{7,8}$ Adrenal myelolipoma equally affects both genders with similar rates and most cases are diagnosed between the $4^{\text {th }}$ and $7^{\text {th }}$ decades of life. They are equally distributed in the right and left adrenal glands but in the majority of cases unilateral, although bilateral lesions are also reported in the literature. ${ }^{8,9}$ Adrenal myelolipomas are usually small $(<4 \mathrm{~cm})$ in diameter, however they can attain very large size. When the size exceeds $8 \mathrm{~cm}$, the term giant myelolipoma is preferred. Akamatsu and Colleagues was found, the largest adrenal myelolipoma (diameter $31 \mathrm{~cm}$, weight $6.0 \mathrm{~kg}$ ). ${ }^{10}$ In our case report, it is right adrenal myelolipoma measuring $24 \times 15 \times 12 \mathrm{~cm}$, weighing $2 \mathrm{~kg}$. However as the size increases, they tend to produce local compressive symptoms and occasionally they may also present with non-specific symptoms.

Adrenal myelolipomas are generally hormonally inactive. Some of these tumors coexist with adrenal adenomas, which may hormonally active. ${ }^{9}$ According to Hisamatsu adrenal myelolipomas are associated with Cushing disease, Conn's syndrome and congenital adrenal hyperplasia. Which are caused by 21-hydroxylase deficiency, $17 \alpha$-hydroxylase deficiency and overproduction of dehydroepiandrosterone-sulphate (DHEAS). ${ }^{11,9}$ Usually adrenal myelolipomas are non-functioning; even then complete hormonal evaluation should be done as there are reports of hormonal hypersecretion and also it is important to exclude pheochromocytoma before any surgical intervention. In our case, the level of serum cortisol, urinary metanephrine, nor-metanephrine and serum potassium were not elevated, which confirmed it to be a non-functional adrenal mass. The exact pathogenesis of myelolipoma is still unsettled; however investigators co-relating myelolipoma with various factors like metaplasia of reticulo-endothelial cells adrenal blood capillaries in response to stimuli like necrosis, infection, stress, long term ACTH stimulation or hematopoietic stimulation by chronic anemia, ectopia of myeloid tissue and embolism of bone marrow. ${ }^{12,13}$ Talwalkar and Shaheen are assumed to arise from metaplasia of either groups of choristomatous hematopoietic stem cells that transmigrate during intrauterine life to the developing adrenal gland or adrenal cortical mesenchymal cells. ${ }^{14}$ The Widespread hypothesis suggests that they arise from the zona fasciculate of the adrenal cortex from metaplasia of undifferentiated stromal cells..$^{15}$ Myelolipoma exhibit four distinct clinicopathologic patterns: 
i. Isolated adrenal myelolipoma,

ii. Adrenal myelolipoma with acute hemorrhage,

iii. Extraadrenal myelolipoma,

iv. Myelolipoma associated with other adrenal disease. ${ }^{15}$

In our case, the histopathologic examination of adrenal myelolipoma, an encapsulated tumor comprising predominantly of mature adipocytes with intervening areas of hematopoietic cells comprising of myeloid cells, erythroid cells, megakaryocytes and occasionally lymphocytesand a rim of normal adrenal at the periphery (Figure 4). Adrenal myelolipoma is usually asymptomatic and detected on radiology imaging. They have typical imaging features, which are often diagnostic; however certain other adrenal lipomatous tumors like lipoma, angiomyelolipoma, teratoma and liposarcoma may also present with similar features and should always be considered. ${ }^{16}$ In $90 \%$ of cases diagnosis of myelolipoma is based on imaging, with ultrasonography, CT, and MRI. ${ }^{9}$ On ultrasonography, a myelolipoma with hypoechoic and hyperechoic area is consists of myeloid cells and adipose tissue respectively. Usually, myelolipoma has mixed hyperechoic and hypoechoic areas. ${ }^{15}$ On CT scan is almost diagnostic for adrenal myelolipoma, the appearance of myelolipoma depends on its histological constituent's. Myelolipoma often has a discrete capsule and appears as well-delineated heterogeneous masses with regions of less than-30 Hounsfield units that correspond to lowdensity mature fat. ${ }^{9}$

The MRI evaluation have not much advantage over CT scan, however the myeloid tissue has low and moderate signal intensity in $\mathrm{T} 1$ and $\mathrm{T} 2$ images respectively whereas adipose tissue has high signal intensity in both $\mathrm{T} 1$ and $\mathrm{T} 2$ images. In case a diagnosis has not been confirmed, a fine needle aspiration can be helpful absolutely rule out malignancy. ${ }^{15}$ The management depends basically on the tumor size, according to Seong Cha when the tumor is asymptomatic with size of $4 \mathrm{~cm}$ and smaller, follow-up with CT-scan is recommended. If the tumor is symptomatic, immediate surgery will be carried out, particularly for a large myelolipoma. If not, there may be spontaneous rupture of the tumor with hemorrhage. ${ }^{8}$ According to NIH guidelines, small, asymptomatic, non-functional myelolipoma may be managed conservatively, however giant adrenal myelolipoma should be excised as the high chances of malignancy and life threatening retroperitoneal or intra-tumoral bleeding is there. ${ }^{17}$ Trans-peritoneal open adrenalectomy is recommended, for larger tumors, however the laparoscopic adrenalectomy may be attempted, depending on the surgeon's expertise. The long term prognosis is excellent, if managed appropriately.

\section{Conclusion}

The adrenal mass was detected incidentally when doing ultrasound of abdomen and followed by CT-Scan. Hyperechoic region was observed in USG due to the presence of adipose tissue in adrenal mass. CT-scan with intravenous contrast injection shows heterogeneous area with well-defined margin which clarify the presence of myelolipoma. Here in we describe a case of a young male with giant adrenal myelolipoma detected incidentally. Therefore, it is very significant to diagnose carefully for the planning of therapeutic management.

\section{Acknowledgements}

Corresponding author acknowledge department of endocrine surgery for performing clinical activities and support from other department for valuable outputs.

\section{Conflict of interest}

Author declares that there is no conflict of interest.

\section{References}

1. Gierke E. Above bone marrow tissue in the adrenal gland. Zeiglers Beitr Path Anat 1905;7:311-324.

2. Oberling C. Les formations myelolipomateuses. Bull Ass Fr Etude Cancer. 1929;18:234-246.

3. Daneshmand S, Marcus L Quek. Adrenal Myelolipoma: Diagnosis and Management. Urol J. 2006;3(2):71-74.

4. Lam KY, Lo CY. Adrenal lipomatous tumor: a 30year clinico-pathological experience at a single institution. J Clin Pathol. 2001;54(9):707-712.

5. Brogna A, Scalisi G, Ferrara R, et al. Giant secreting adrenal myelolipoma in a man: a case report. J Med Case Rep. 2011;5:298.

6. Boudreaux D, Waisman J, Skinner DG, et al. Giant adrenal myelolipoma and testicular interstitial cell tumor in a man with congenital 21-hydroxylase deficiency. Am J Surg Pathol. 1979;3(2):109-123.

7. Kammen BF, Elder DE, Franker DL, et al. Extraadrenal myelolipoma: MR imaging findings. AJR Am J Roentgenol. 1998;171(3):721-723.

8. Cha SJ, Shin SY, Kim MK, et al. Myelolipoma of both adrenal glands. Koran J Urol. 2011;52(8):582-585.

9. Daneshmand S, Quek ML. Adrenal myelolipoma: diagnosis and management. J Urol. 2006;3(2):71-74.

10. Akamatsu H, Koseki M, Nakaba H, et al. Giant adrenal myelolipoma: report of a case. Surg Today. 2004;34(3):283-285.

11. Hisamatsu H, Sakai H, Tsuda S, et al. Combined adrenal adenoma and myelolipoma in a patient with Cushing's syndrome: case report and review of the literature. Int J Urol. 2004;11(6):416-418.

12. Mukherjee S, Pericleous S, Hutchins RR, et al. Asymptomatic giant adrenal myelipoma. Urol J. 2010;7(1):66-68

13. Wilhelmus JL, Schrodt GR, Alberhasky MT, et al. Giant adrenal myelolipoma: case report and review of the literature. Arch Pathol Lab Med. 1981;105(10):532-535.

14. Talwalkar SS, Shaheen SP. Extra-adrenal myelolipoma in the renal hilum: a case report and review of the literature. Arch Pathol Lab Med. 2006;130(7):1049-1052.

15. Rao P, Kenney PJ, Wagner BJ, et al. Imaging and pathologic features of myelolipoma. Radio Graphics 1997;17(6):1373-1385.

16. Lam KY, Lo CY. Adrenal lipomatous tumor: a 30year clinico-pathological experience at a single institution. J Clin Pathol 2001;54(9):707-712.

17. NIH state-of-the-science statement on the management of the clinically inapparant adrenal masse ("incidentaloma"). NIH Consens State Sci Statements. 2002;19(2):1-25 